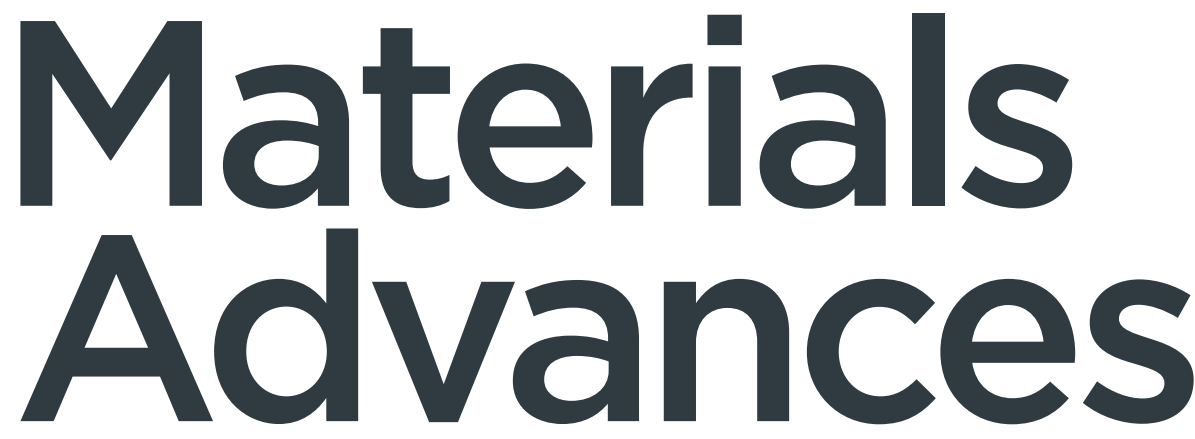

Number 6

21 March 2022

Pages 2601-2948

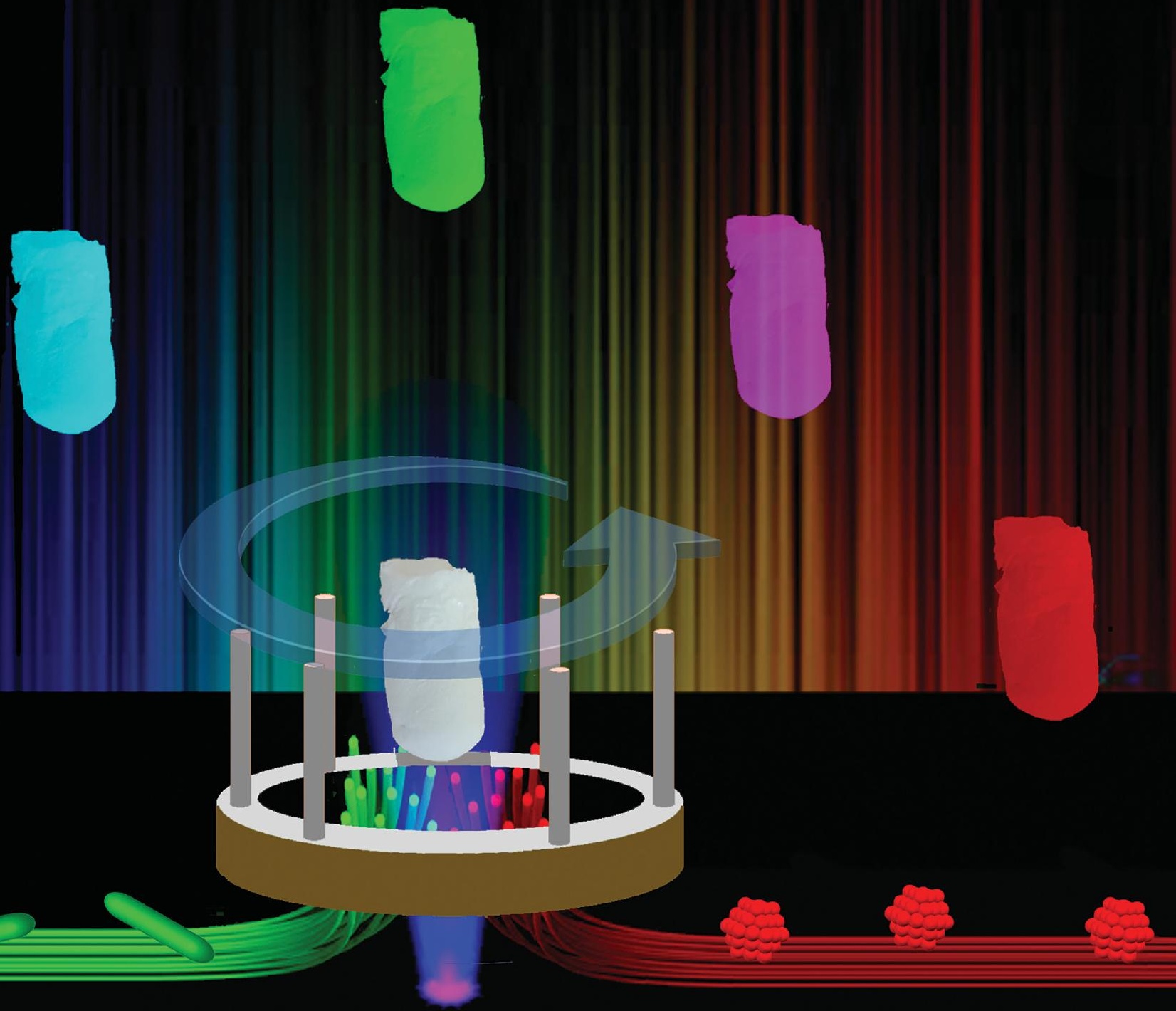

ISSN 2633-5409 OF CHEMISTRY

\section{PAPER}

Karen Lozano et al.

Color tunable aerogels/sponge-like structures developed from fine fiber membranes 
Check for updates

Cite this: Mater. Adv., 2022, 3,2716

Received 20th October 2021, Accepted 2nd February 2022

DOI: $10.1039 / \mathrm{d} 1 \mathrm{ma} 00946$

rsc.li/materials-advances

\title{
Color tunable aerogels/sponge-like structures developed from fine fiber membranes
}

\author{
Alexa Villarreal, $\dagger^{a}$ Raul Barbosa, $\dagger^{a}$ Saptasree Bose, ${ }^{a}$ Bhupendra B. Srivastava, (iD b \\ Victoria Padilla-Gainza ${ }^{a}$ and Karen Lozano*a
}

\begin{abstract}
The development of macroscopic aerogels from 1D systems, such as nanofibers, has resulted in a novel pathway to obtain porous and lightweight architectures. In this work, bright green, red, and tunable color emitting aerogels were obtained with luminescent nanofibers as the precursor system. A simple, low cost, and environmentally friendly process is followed where luminescent fillers are encapsulated within fibers which were subsequently freeze-dried to form 3D aerogels and sponge-like structures. Moreover, the aerogels/sponge-like structures show higher photoluminescence intensity than the fiber mats due to an increase of porosity which provides higher and direct interaction with the fillers and therefore an efficient light absorption resulting in higher luminescence. Manganese doped zinc germanate $\left(\mathrm{Mn}: \mathrm{Zn}_{2} \mathrm{GeO}_{4}\right)$ nanorods and chromium doped zinc gallate $\left(\mathrm{Cr}\right.$ : $\left.\mathrm{ZnGa}_{2} \mathrm{O}_{4}\right)$ nanoparticles were used as the source of green and red emissions respectively. By precisely adjusting the stoichiometric ratios of nanorods and nanoparticles within the nanofibers, a broad spectrum output is obtained from the final aerogels. We foresee that these types of photoluminescent aerogels have promising potential applications in a variety of fields such as display devices, solid-state lighting, sensors, etc.
\end{abstract}

\section{Introduction}

Aerogels are solid, lightweight, and highly porous materials. Their solid porous 3D network structure, high specific surface area, low density, and unique chemical, thermal and optical properties make them suitable candidates for a variety of applications in the fields of catalysis, energy storage, and sensors. ${ }^{1-4}$ Recently, luminescent aerogels have attracted much attention as they open new opportunities in the design of optically tunable materials with spontaneous adjustable color emissions, for the purpose of solid-state lighting and other optical applications. ${ }^{1,5,6}$ The design of fluorescent aerogels is based on the appropriate interaction between fluorescent chromophores and the aerogel framework to achieve bright persistent luminescence. ${ }^{7}$ Traditional fluorescent materials such as quantum dots, perovskite nanocrystals, metal-organic frameworks, organic fluorescent dyes, lanthanide-doped glass, and ceramic or crystalline phosphors are promising luminescent materials due to their superior optical properties including intense luminescence, sharp and stable emission peaks, and

\footnotetext{
${ }^{a}$ Mechanical Engineering Department, University of Texas Rio Grande Valley, 1201 W. University Dr., Edinburg, Texas 78539, USA.

E-mail: karen.lozano@utrgv.edu

${ }^{b}$ Department of Chemistry, University of Texas Rio Grande Valley, 1201 W. University Dr., Edinburg, Texas 78539, USA

$\dagger$ Alexa Villarreal and Raul Barbosa contributed equally to this work.
}

high quantum yield. ${ }^{7-10}$ Two distinct strategies for tuning the luminescence of chromophore-doped aerogels include: changing the dopant type/nature and/or varying the doping concentration. For example, in 2016, Niederberger et al. synthesized a $\mathrm{Eu}^{3+}$ and $\mathrm{Tb}^{3+}$ doped $\mathrm{Y}_{2} \mathrm{O}_{3}$ luminescent aerogel with adjustable spectral emission from red to green. ${ }^{11}$ Srivastava et al. have also reported on the variation of the doping concentration to produce tunable emission ranging from white to red to green. ${ }^{12}$ Similarly, veinot et al. fabricated hydrophilic silicon nanocrystals of various sizes and surface groups, and incorporated them into tetramethyl orthosilicate to produce aerogels with different levels of transparency. ${ }^{6}$ In 2018, Ren et al. produced $\mathrm{Al}_{2} \mathrm{O}_{3}-\mathrm{Eu}^{3+}$ luminescent aerogels which can be tailored to emit pink to green colors by varying the doping concentration of $\mathrm{Eu}^{3+} \cdot{ }^{13}$ In 2020, Yang et al. offered a facile technique to prepare a white light emitting aerogel with the modulation of the stoichiometric ratio of lanthanides, thymidine, and carbon dots that display reversible stimuli-responsive properties. ${ }^{1}$ However, fluorescent composite aerogels processed by freeze drying or a critical point drying treatment suffer from insufficient interaction resulting in mechanical instability and aggregation of the chromophore, which can induce quenching of fluorescence intensity. ${ }^{7}$ Recently, luminescent nanofibers have emerged as an exciting luminescent material with paramount potential due to their excellent flexibility, high specific surface area, and light weight properties. Notably, 3D porous structures like aerogels can be synthesized using nanoscale 
building blocks such as polymeric nanofibers; the resulting aerogels are highly elastic, flexible and environmentally friendly. ${ }^{14}$ However, producing 3D scaffolds via the layer-by-layer deposition of luminescent nanofibers during electrospinning reduces the possibility to add desired scalable pores within the aerogels and they often exhibit mechanical instability. ${ }^{15-17}$ Ding and Greiner independently initiated a facile strategy to produce ultralight nanofiber-based aerogels by wetting and cutting long electrospun fibers until a thorough dispersion of the fibers formed followed by freezing the solution and subsequently placing it in a freezedryer. ${ }^{15,18-26}$ Despite investigating numerous approaches to produce short nanofibers for nanofiber-based aerogels, the method of mechanically cutting and dispersing continuous long fibers is considered as the most straightforward and simple technique in this newly emerging field of research. ${ }^{15,27-31}$

In this study, lightweight bright luminescent aerogels were synthesized from fluorescent polyvinyl alcohol (PVA) fibers. PVA is a biocompatible, chemically and mechanically stable polymer. In this process, luminescent polymer nanofibers were prepared using in-house synthesized green luminescent manganese doped zinc germanate ( $\left.\mathrm{Mn}: \mathrm{Zn}_{2} \mathrm{GeO}_{4}, \mathrm{ZGOM}\right)$ nanorods ${ }^{32,33}$ and red luminescent chromium doped zinc gallate $\left(\mathrm{Cr}: \mathrm{ZnGa}_{2} \mathrm{O}_{4}\right.$, ZGOC) nanoparticles with sub-10 $\mathrm{nm} \operatorname{size}^{34-36}$ as the doping agents. The nanofibers were produced using the forcespinning ${ }^{\mathbb{R}}$ method instead of the electrospinning method to avoid the use of electric fields and organic solvents with specific dielectric constant, which is a primary requirement for the electric field driven electrospinning method. Moreover, the centrifugal spinning process was used which resulted in high yield and as a result a lower cost process. For the freeze-drying process, deionized water and 1,4-dioxane, as non-polar aprotic solvents, were selected as the dispersion media. The influence of the dispersion medium on the morphology and luminescence of the final structures was studied. PVA is known for its solubility in water, and nanofiber integrity was lost after the homogenization procedure, however, the fillers remained well dispersed and distributed, and neither sedimentation nor aggregation was observed. Upon freeze-drying, the final structure portrayed aerogel characteristics, and microscopy images revealed a porous solid network with porous morphology dependent upon filler morphology. As for the systems subjected to the dioxane medium, nanofibers were not dissolved and the ultimate 3D architecture revealed a disordered porous arrangement highly dependent on nanofiber entanglement resembling a sponge-like structure. Moreover, the resulting aerogels/sponges show higher luminescence when compared to their precursor nanofiber mats. The modulation of the stoichiometric ratio of red nanoparticles (NPs) and green nanorods (NRs) within the fiber matrix offers a final aerogel product with broad luminescence ranging from red to green with promising potential applications in a wide variety of fields.

\section{Experimental}

\subsection{Materials}

Zinc nitrate hexahydrate $\left(\mathrm{Zn}\left(\mathrm{NO}_{3}\right)_{2} \cdot 6 \mathrm{H}_{2} \mathrm{O}, 98 \%\right)$, gallium nitrate hydrate $\left(\mathrm{Ga}\left(\mathrm{NO}_{3}\right)_{3} \cdot x \mathrm{H}_{2} \mathrm{O}, 99.9 \%\right)$, chromium nitrate nonahydrate
$\left(\mathrm{Cr}\left(\mathrm{NO}_{3}\right)_{3} \cdot 9 \mathrm{H}_{2} \mathrm{O}, 99 \%\right)$, germanium oxide $\left(\mathrm{GeO}_{2}, 99.99 \%\right)$, manganese(II) nitrate tetrahydrate $\left(\mathrm{Mn}\left(\mathrm{NO}_{3}\right)_{2} \cdot 4 \mathrm{H}_{2} \mathrm{O}, 97 \%\right)$, urea and ammonium hydroxide solution (28.0-30.0\% $\mathrm{NH}_{3}$ basis), and 1,4-dioxane (99.8\%) were purchased from Sigma Aldrich. Poly(vinyl alcohol) (27-96 medium hydrolyzed grade) was purchased from Kuraray Poval ${ }^{\mathrm{TM}}$. Deionized (DI) water $(25 \mathrm{M} \Omega \mathrm{cm}$ ) was produced from a Smart2Pure water purification system. All the chemicals were of analytical grade reagents and used directly without further purification. Persistent red luminescent sub-10 nm water dispersible ZGOC nanoparticles were synthesized following a simple and facile synthesis route. ${ }^{36}$ The water dispersible ZGOM nanorods were produced using the hydrothermal method described in the literature. ${ }^{32}$

\subsection{Synthesis of luminescent PVA fine fibers}

The $100 \%$ pure green and red fibers were prepared by incorporating $56 \mathrm{mg}$ each of ZGOM and ZGOC, respectively, in $10 \mathrm{~mL}$ of DI water, followed by 3 minutes of homogenization using a probe sonicator (Sonic Dismembrator, Model 120, Fisherbrand $\left.^{\mathrm{TM}}\right)$. Subsequently, $1.11 \mathrm{~g}$ of PVA was added to the previously prepared homogenized solution and placed in an oil bath for $1 \mathrm{~h}$, at $75{ }^{\circ} \mathrm{C}$, with a magnetic stirrer. The solution was then taken out of the oil bath and stirred overnight at room temperature.

The fibers were produced by adding $2 \mathrm{~mL}$ of the prepared solution in the spinneret of a Cyclone ${ }^{\mathrm{TM}} \mathrm{L}-1000 \mathrm{M}$, purchased from Fiberio Technology Corporation (McAllen, USA). Then it was spun from 5000 to $7000 \mathrm{rpm}$ to allow the solution to exit through the spinneret's orifices. The solution was ejected from both ends of the spinneret through 30 gauge needles, and was deposited onto equally distanced vertical pillars, thus creating fine fibers which were then collected with a $10 \times 10 \mathrm{~cm}$ collector after each cycle. Fibers were covered and stored in a low moisture environment. The ZGOM and ZGOC encapsulated PVA fine fibers are referred to as green PVA fibers (G-PF) and red PVA fibers (R-PF).

Four other composite luminescent fibers using different green nanorods and red nanoparticle concentrations were prepared following the method described above: (1) $90 \%$ red $+10 \%$ green; (2) $80 \%$ red $+20 \%$ green; (3) $60 \%$ red $+40 \%$ green and (4) $40 \%$ red $+60 \%$ green.

\subsection{Synthesis of luminescent aerogels}

The fiber mat $(40 \mathrm{mg})$ was cut into $1 \times 1 \mathrm{~cm}^{2}$ pieces and dispersed in $4 \mathrm{~mL}$ of DI water and subjected to multiple cycles of homogenization performed at intervals of 10 seconds. Two to three pieces of the precut mats were placed in the DI water container after each cycle and homogenization was continued using a PRO Scientific Bio-Gen PRO200 homogenizer to obtain a uniform fiber dispersion within the medium. The solution was then poured into a plastic mold and frozen at $-82{ }^{\circ} \mathrm{C}$ in a freezer for 24 hours at a controlled temperature rate. Thereafter, the frozen system was placed in a freeze dryer for 48 hours to remove the solvent through sublimation. A 3D porous structure was obtained (Scheme 1). For the preparation of the green, red, and composite aerogels: ZGOM encapsulated PVA (green), ZGOC 


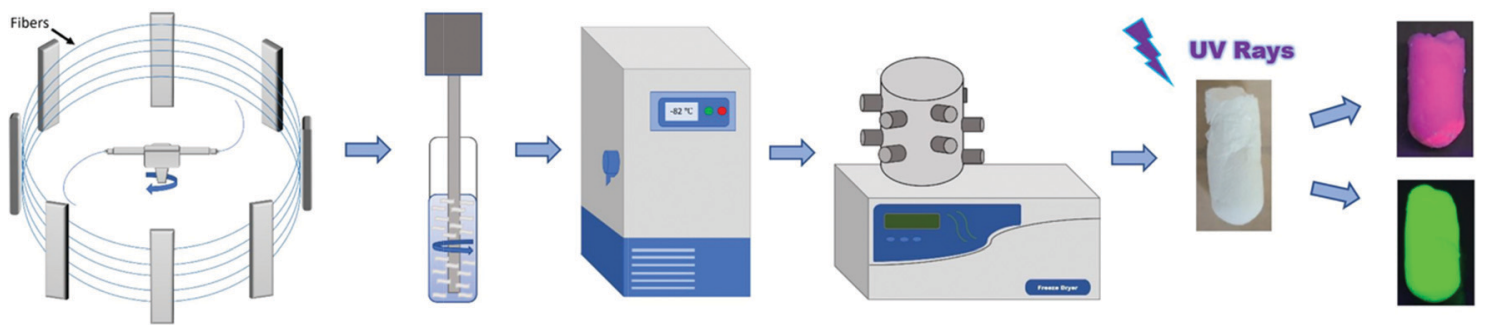

Scheme 1 Schematic diagram for the synthesis of aerogels.

encapsulated PVA (red) and different ratios of ZGOM:ZGOC encapsulated PVA (composite) fibers were used as the precursors, respectively. The same procedure was followed to prepare the 1,4-dioxane based sponge-like 3D porous structure. It was seen during synthesis that unlike the system subjected to the waterbased medium, the system subjected to the dioxane medium exhibited an opaque appearance, resulting from the prevailing nanofibers just suspended in the liquid and dispersed throughout the solution rather than fiber dissolution as happened with the water medium.

\subsection{Instrumentation}

To quantify the developed luminescent nanoparticles, powder X-ray diffraction (XRD) data were collected using a scanning mode of $2 \theta$ with a scanning step size of $0.04^{\circ}$ and a scanning rate of $2.0^{\circ} \mathrm{min}^{-1}$. X-ray photoelectron spectroscopy (XPS) was carried out using a Thermo scientific K-alpha XPS with $180^{\circ}$ double focusing hemispherical analyzer with 128 channel detectors. The accuracy of the binding energy (BE) was $0.1 \mathrm{eV}$ as calibrated by $\mathrm{Cu} 3 \mathrm{P}_{3 / 2}(75.1 \mathrm{eV})$ and $\mathrm{Cu} 2 \mathrm{P}_{3 / 2}(932.7 \mathrm{eV})$ reference peaks. Survey scan spectra of the fibrous mats were taken with 10 scans at a surface scan spot of 300 microns. Morphological analysis of the fine fibers and solid aerogels was carried out using a scanning electron microscope (SEM) (Sigma VP; Carl Zeiss, Jena, Germany). Thermogravimetric analysis was completed using a NETZSCH TG 209 at a rate of $10{ }^{\circ} \mathrm{C} \mathrm{min}{ }^{-1}$ under $\mathrm{N}_{2}$. The excitation and emission spectra of the as-prepared fine fibers and aerogels were recorded using an
Edinburgh Instruments FLS980 fluorimeter system equipped with a xenon (Xe) lamp, as an excitation source, and a TE-cooled photo-multiplier tube (Hamamatsu, Model R928P) for emission detection. All emission spectra were corrected for determining the spectral sensitivity of the system and detector, as well as the intensity variation in the Xe light source using a reference diode. Samples $(1 \times 1 \mathrm{~cm})$ were prepared and placed in a Thermo Nicolet Nexus ${ }^{\mathrm{TM}} 470$ FT-IRESP Fourier Transform Infrared (FTIR) machine. After removing the background spectrum, the specimens were scanned in a $400-4000 \mathrm{~cm}^{-1}$ range with a resolution of $4 \mathrm{~cm}^{-1}$.

\section{Results and discussion}

\subsection{Structural and morphological characterization of bare ZGOM and ZGOC nanoparticles}

The nanoparticles were synthesized following the aforementioned procedure. Fig. 1 represents the XRD patterns of ZGOM nanorods and ZGOC nanoparticles in the powder form. The results adhere to the respective standard patterns of $\mathrm{Zn}_{2} \mathrm{GeO}_{4}$ (JCPDS file no. 11-0687) and $\mathrm{ZnGa}_{2} \mathrm{O}_{4}$ (JCPDS file no. 86-0415), indicating the formation of pure phased compounds, and also the presence of sharp peaks suggests expected crystallinity.

The FESEM images of the hydrothermally synthesized ZGOM nanorods and ZGOC nanoparticles are shown in Fig. 2a and b, respectively. The morphology of the ZGOM shows the formation of nanorods with smooth surfaces. The majority of NRs show
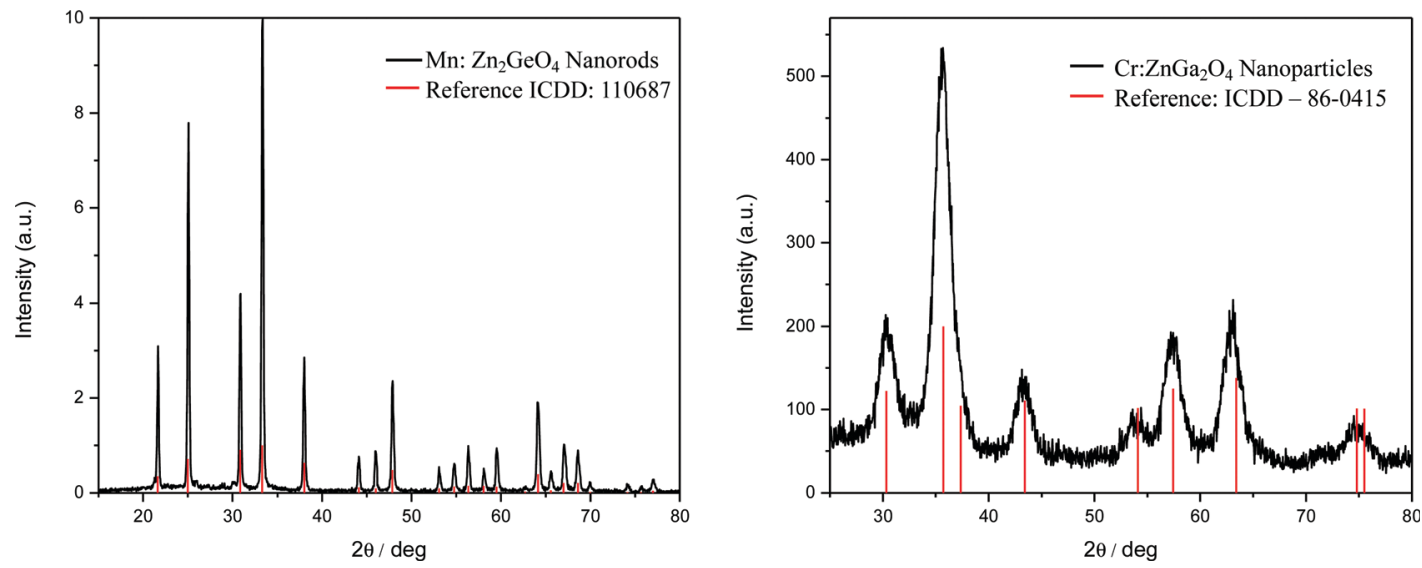

Fig. 1 Powder XRD patterns of ZGOM nanorods and ZGOC nanoparticles. 
lengths of $1-3 \mu \mathrm{m}$ with $200-300 \mathrm{~nm}$ diameter. ${ }^{32}$ The FESEM image of ZGOC clearly demonstrates the formation of spherical nanoparticles ranging in diameter from 10-20 nm.

\subsection{Characterizations of G-PF and R-PF fibers}

The G-PF and R-PF fine fibers show smooth, long, continuous fiber morphology without aggregation of nanorods or nanospheres (Fig. 3a and b). Moreover, there are no beads present in the developed fibers, and the fiber mats are highly flexible. The digital photographs of the bright green and red fiber mats are shown in the inset of Fig. 3. Elemental mapping was performed (Fig. 3c and d) on the pure green and pure red fibers proving the presence and uniform distribution of the green nanorods and red nanoparticles within the PVA fiber showing a concentration of $5 \% \mathrm{Mn}, 18 \% \mathrm{Zn}, 16 \% \mathrm{Ge}$, and $61 \% \mathrm{O}$ for the green nanorods and $15 \% \mathrm{Cr}, 10 \% \mathrm{Zn}, 11 \% \mathrm{Ga}$, and $64 \% \mathrm{O}$ for the red nanoparticles.

\subsection{Morphological characteristics of the fluorescent fiber-based aerogels (FA)}

It has been reported that the dispersion medium (i.e. water, ethanol, camphene, tert-butyl alcohol, toluene, 1,4-dioxane) plays an important role in the physical, mechanical, and chemical properties of freeze-dried aerogels. ${ }^{15}$ The green and red aerogels were prepared via the freeze-drying method using two different dispersion liquids: water and 1,4-dioxane. The porous morphology shown in the green and red water-based aerogels is derived from the formation of ice crystals during the process of freezing the solution. Fig. 4a-c depict the SEM images of the aerogel-like structure that resulted from treatment under the water medium, while Fig. 4d illustrates the sponge-like structure that resulted from exposure to the dioxane medium. It is worth noting that Fig. 4a shows a material made from 100\% G-PF where green luminescent nanorods were used, and the material shown in Fig. $4 \mathrm{~b}$ is composed of $100 \%$ R-PF which contains spherical nanoparticles. The morphology of the pores (size and shape) is clearly affected by the nanoparticles and nanorods used within the precursor fiber systems. Fig. 4a exhibits elongated pores, while Fig. $4 \mathrm{~b}$ displays circular pores. When combined (G-PF/R-PF composite fiber mats), the final product demonstrates traits of both nanomaterials, round pores with larger diameters, and is slightly elongated (i.e. Fig. 4c). The role played by the ZGOC spherical NPs and ZGOM nanorods in the systems subjected to water medium dominates the ultimate shape of the pores within the aerogel structure since the nanofiber structure was lost.
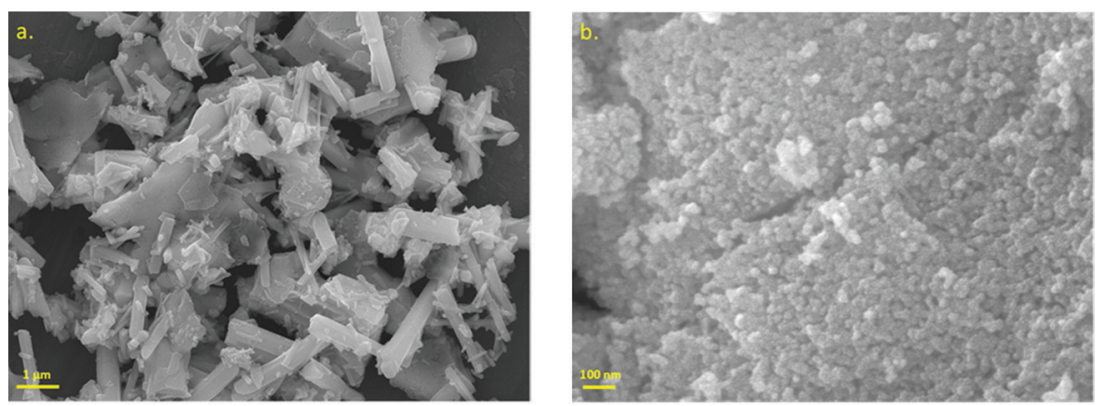

Fig. 2 FESEM images of (a) ZGOM and (b) ZGOC nanoparticles.
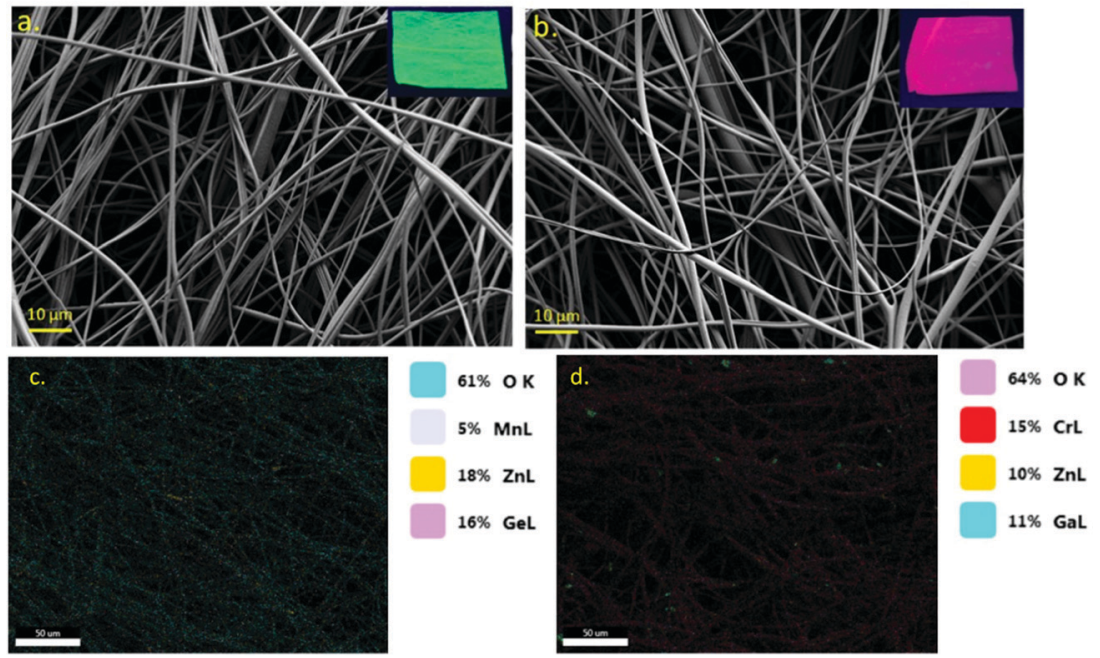

Fig. 3 (a) FESEM image of green nanoparticle encapsulated G-PF fibers. (b) FESEM image of red nanoparticle encapsulated R-PF fibers; (c) elemental mapping of G-PF and (d) R-PF. 


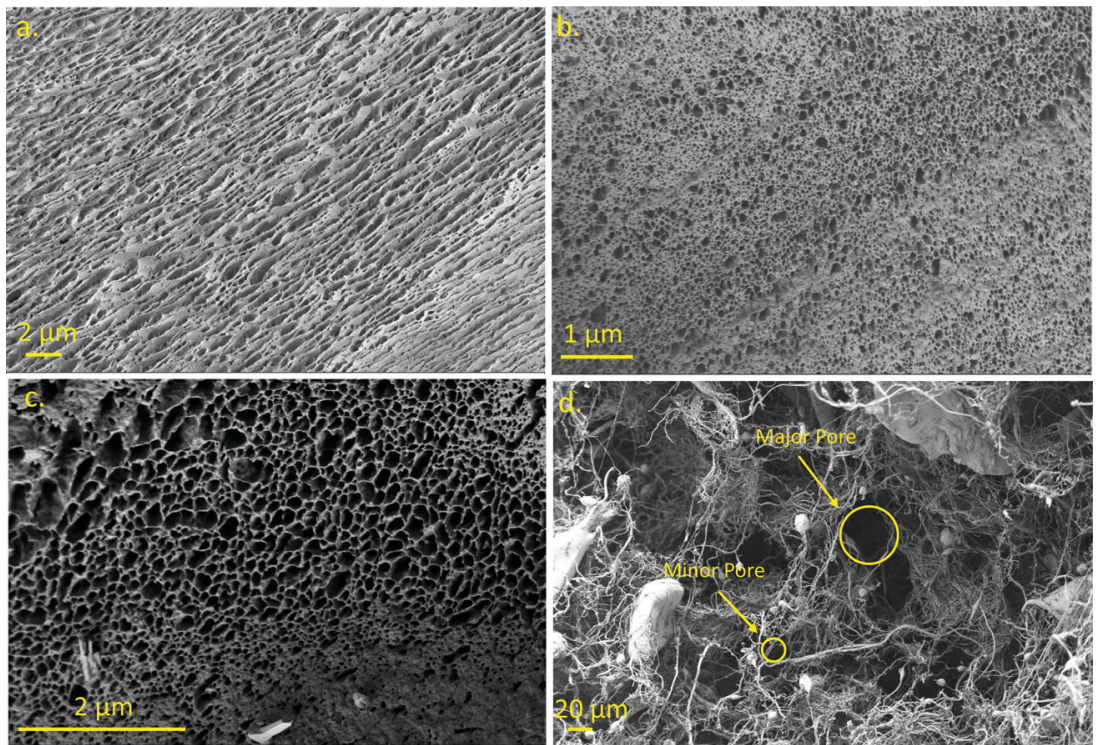

Fig. 4 FESEM images of (a) green aerogels produced from G-PF samples using water as dispersion medium; (b) red aerogels produced from R-PF samples using water as the dispersion medium; (c) composite aerogels produced from the composite fiber (encapsulating both green nanorods and red nanorods/nanoparticles) using water as the dispersion medium; and (d) red sponge-like structure using 1,4-dioxane as the dispersion medium.

The NPs and NRs act as templates for the growth of ice crystals. During the freezing phase, anisotropy phase separation occurs between ice and the polymer/fillers, forming intercalated ice crystals around the PVA and respective NP/NR walls. Freeze drying removes ice from the system and leaves pores shaped according to the composition of the system.

As for the system developed using dioxane as the medium, SEM images show a porous, sponge-like fibrous network composed of preserved entangled nanofibers where a highly disordered pore structure is observed (Fig. 4d).

The sponge-like 3D porous network produced using 1,4dioxane as the dispersion medium (Fig. 4d) shows a very different morphology when compared to the water-based aerogels. The removal of the ice crystals by sublimation resulted in a nanofibrous framework characterized by a large variation in the pore size and morphology. The porous network is less compact in nature and the pores are not homogeneous as compared to the water-based aerogels. Pore size and porosity can be tailored not only by altering processing parameters such as freezing rate, fiber loading, and solvent, but also by the morphology of the encapsulated particles within the starting fibers.

\subsection{Fourier transform infrared spectroscopy (FTIR)}

Fig. 5 shows the FTIR spectra for the green, red and the fine fibers of the composite along with the aerogels made from the fibers. The infrared spectra for the region $400-4000 \mathrm{~cm}^{-1}$ correspond to the most prominent features of the PVA. The main peaks of PVA were observed at $3287 \mathrm{~cm}^{-1}, 2919 \mathrm{~cm}^{-1}$, $1711 \mathrm{~cm}^{-1}, 1424 \mathrm{~cm}^{-1}, 1325 \mathrm{~cm}^{-1}, 1084 \mathrm{~cm}^{-1}$, and $837 \mathrm{~cm}^{-1}$. These peaks are ascribed to the $\mathrm{O}-\mathrm{H}$ stretching vibration of the hydroxy group, $\mathrm{C}-\mathrm{H}$ stretching, $\mathrm{C}=\mathrm{O}$ stretching, $\mathrm{C}-\mathrm{H}$ bending, $\mathrm{CH}_{2}$ asymmetric stretching vibration, $\mathrm{C}=\mathrm{O}$ carbonyl stretch,
$\mathrm{C}-\mathrm{H}$ bending vibration of $\mathrm{CH}_{2}, \mathrm{C}-\mathrm{O}$ (acetyl) stretching, and $\mathrm{C}-\mathrm{C}$ stretching vibration, respectively. ${ }^{37,38}$ It is important to note that the normalized FTIR peaks of the fibers and aerogels are in very similar positions.

\subsection{X-Ray photoelectron spectroscopy (XPS)}

To confirm the presence of $\mathrm{Mn}: \mathrm{Zn}_{2} \mathrm{GeO}_{4}$ nanorods within the aerogel matrix, X-ray photoelectron spectroscopy (XPS) analysis was performed. The XPS spectrum of the green aerogel revealed that the sample is comprised of $\mathrm{Zn}, \mathrm{Ge}, \mathrm{Mn}, \mathrm{O}$, and $\mathrm{C}$ (Fig. 6a). Fig. $6 \mathrm{~b}$ shows the representative peaks of the $\mathrm{Zn}^{2+}$ oxidation states at 1022 and $1045 \mathrm{eV}$, corresponding to $\mathrm{Zn} 2 \mathrm{p}_{3 / 2}$ and $\mathrm{Zn}$ $2 p_{1 / 2}$ respectively. ${ }^{39}$ Similarly, Fig. $6 \mathrm{c}$ exhibits the characteristic Ge $3 \mathrm{~d}$ peak at $31.5 \mathrm{eV}$ which is ascribed to the $\mathrm{Ge}-\mathrm{O}$ bonding in the form of $\mathrm{Ge}^{4+} \cdot{ }^{39,40}$ Fig. $6 \mathrm{~d}$ displays the XPS spectrum of Mn $2 \mathrm{p}$ with two peaks at 642 and $655 \mathrm{eV}$, which could be due to Mn $2 \mathrm{p}_{3 / 2}$ and $\mathrm{Mn} 2 \mathrm{p}_{1 / 2}$ levels, respectively. The spin energy separation of $\sim 13 \mathrm{eV}$, is a characteristic of the $\mathrm{Mn}^{2+}$ oxidation state. ${ }^{39,41}$ The presence of $\mathrm{C}$ and $\mathrm{O}$ is confirmed from the survey spectrum.

XPS was carried out for the red aerogel to check the presence of red luminescent $\mathrm{Cr}: \mathrm{ZnGa}_{2} \mathrm{O}_{4}$ nanoparticles within the aerogel network. Fig. 6e shows the survey XPS spectrum confirming the presence of $\mathrm{C}, \mathrm{O}, \mathrm{Ga}, \mathrm{Zn}$, and $\mathrm{Cr}$. The XPS spectrum in Fig. 6f shows two characteristic peaks of $\mathrm{Zn}^{2+}$ at $1022\left(2 \mathrm{p}_{3 / 2}\right)$ and $1045 \mathrm{eV}\left(2 \mathrm{p}_{1 / 2}\right){ }^{42}$ The XPS spectrum of $\mathrm{Ga}$ is shown in Fig. $6 \mathrm{~g}$ having a characteristic peak at $18.8 \mathrm{eV}$ which is attributed to $\mathrm{Ga} 3 \mathrm{~d} .{ }^{43}$ The presence of $\mathrm{Cr}$ has been displayed in Fig. $6 \mathrm{~h}$ where a peak has been found at $585 \mathrm{eV}$ corresponding to $\mathrm{Cr} 2 \mathrm{p}_{1 / 2} \cdot{ }^{44}$ The presence of $\mathrm{C}$ and $\mathrm{O}$ is confirmed from the survey spectrum. All of the binding energy values and gaps between individual elements of the luminescent green nanorods and red nanoparticles (which were encapsulated in the 
a.

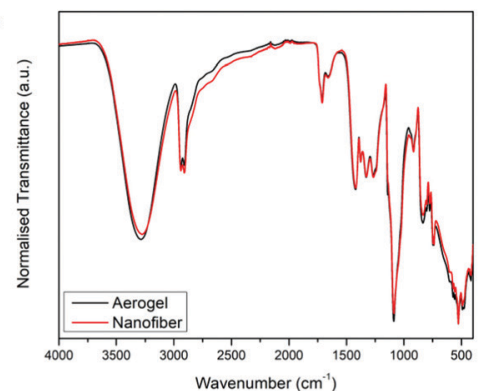

b.

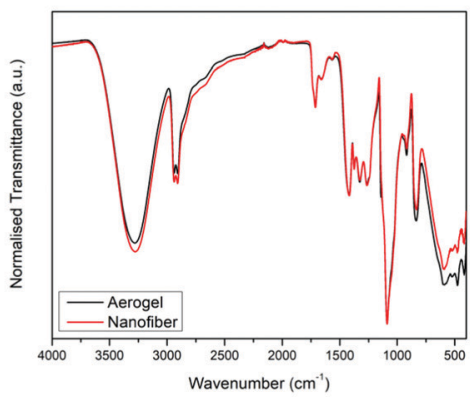

c.

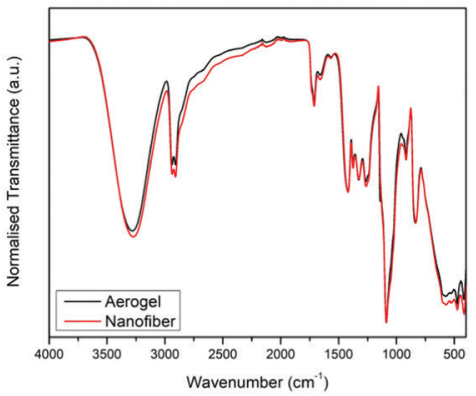

Fig. 5 FTIR spectra for (a) pure green; (b) pure red and (c) composite water-based aerogels.
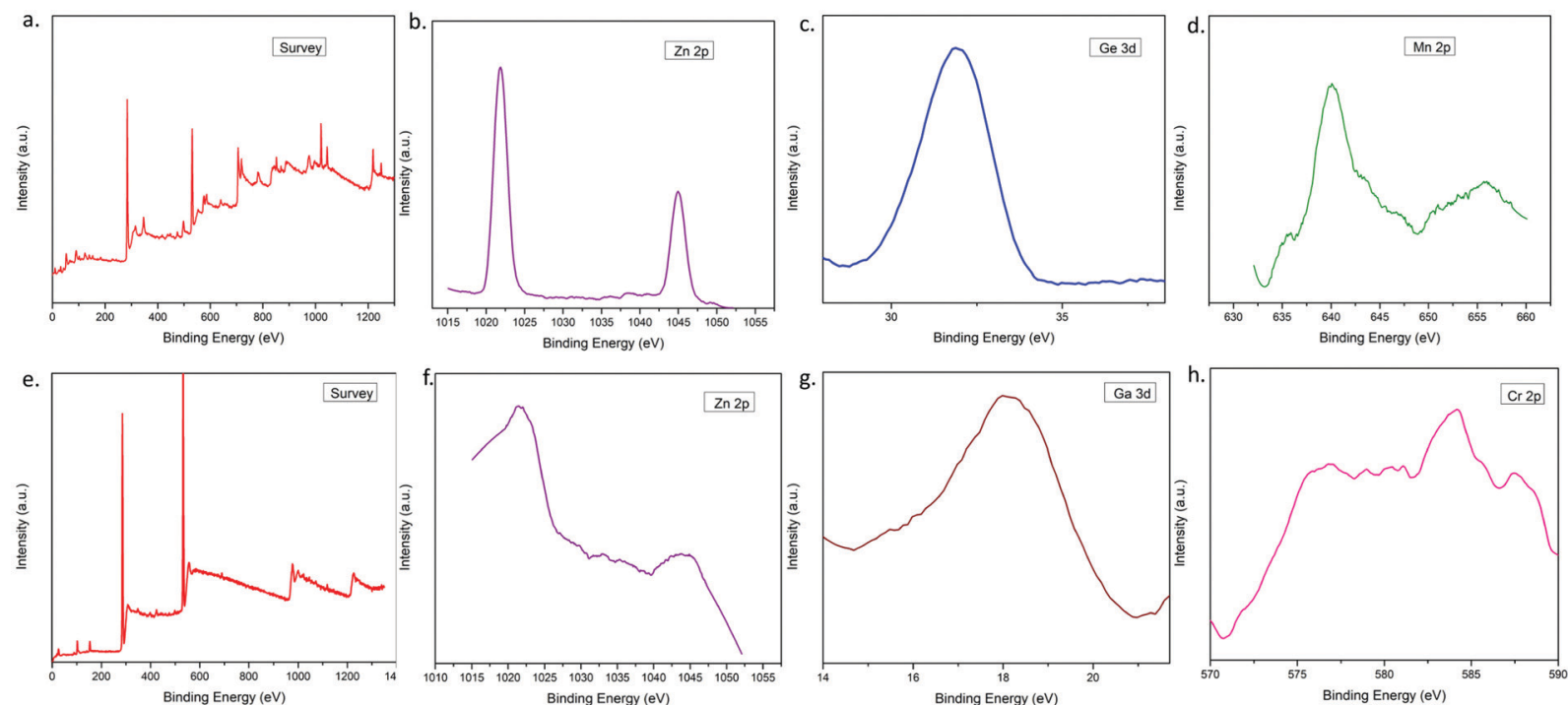

Fig. 6 The XPS spectra of the pure green water-based aerogel (upper panel): (a) survey scan spectrum; (b) the Zn 2p spectrum; (c) the Ge 3d spectrum; (d) the Mn $2 \mathrm{p}$ spectrum and the pure red water-based aerogel (lower panel): (e) survey scan; (f) $\mathrm{Zn} \mathrm{2p;} \mathrm{(g)} \mathrm{Ga} \mathrm{3d;} \mathrm{and} \mathrm{(h)} \mathrm{Cr} 2 \mathrm{p}$.

precursor nanofibers) matched well with the reference values, confirming the existence and phase purity of the luminescent particles within the aerogel.

\subsection{Thermogravimetric analysis (TGA)}

TGA analysis was carried out for the developed fiber and aerogel systems to evaluate their thermal stability. As expected, negligible changes are observed for the systems when analyzed as fibers or aerogels. Thermograms are shown in Fig. 7a and b. For the green fibers and green aerogel, the first weight loss of $7 \%$ occurs at approximately $100{ }^{\circ} \mathrm{C}$ due to the evaporation of adsorbed water. ${ }^{45}$ After this, 65\% weight loss occurred in the temperature range of 250 to $350{ }^{\circ} \mathrm{C}$ which involves the dehydration of PVA to polyene. Notably, in the case of the green fibers the complete formation of polyene was observed at $352{ }^{\circ} \mathrm{C}$, whereas the corresponding green aerogel shows a similar transition at $342{ }^{\circ} \mathrm{C}$. The dehydrated PVA was thermally decomposed by chain scission above $476{ }^{\circ} \mathrm{C}$. The red fiber and red aerogel show a similar loss ( $7 \%$ weight loss) of adsorbed water at $100{ }^{\circ} \mathrm{C}$. The dehydration of PVA ranged from $260-370{ }^{\circ} \mathrm{C}$ which is associated with a $70 \%$ weight loss. Thereafter, thermal decomposition of polyene via chain degradation occurred from $370-480{ }^{\circ} \mathrm{C}$.

\subsection{Photoluminescence (PL) measurements}

Fig. 8 shows the photoluminescence (PL) properties of the fibers and aerogels. The digital photographs (Fig. 8a) clearly exhibit the appearance of the fluorescent fibers and aerogels under UV light, indicating the successful tuning of color emission depending on the stoichiometric changes of the encapsulated nanoparticles within the fiber matrix. PL emission spectra for green and red aerogels (Fig. 8b and c) show intense emission peaks at 535 and $695 \mathrm{~nm}$, respectively, under UV light (254 nm) excitation. The unaltered emission peaks were observed for the green and red nanoparticles, as well as for the fine PVA fibers, which encapsulate the nanoparticles ${ }^{32,33,36}$ demonstrating the conservation of the original emission bands of the nanoparticles even after the covalent linkage of the luminescent fibers in the final 3D network of the aerogels. The green peak at $535 \mathrm{~nm}$ is attributed to the radiative ${ }^{4} \mathrm{~T}_{1}(4 \mathrm{G})$ $\rightarrow{ }^{6} \mathrm{~A}_{1}(6 \mathrm{~S})$ transition due to parity forbidden $\mathrm{d} \rightarrow \mathrm{d}$ transition of the $\mathrm{Mn}^{2+}$ ion in ZGOM NRs, encapsulated in the fine PVA 

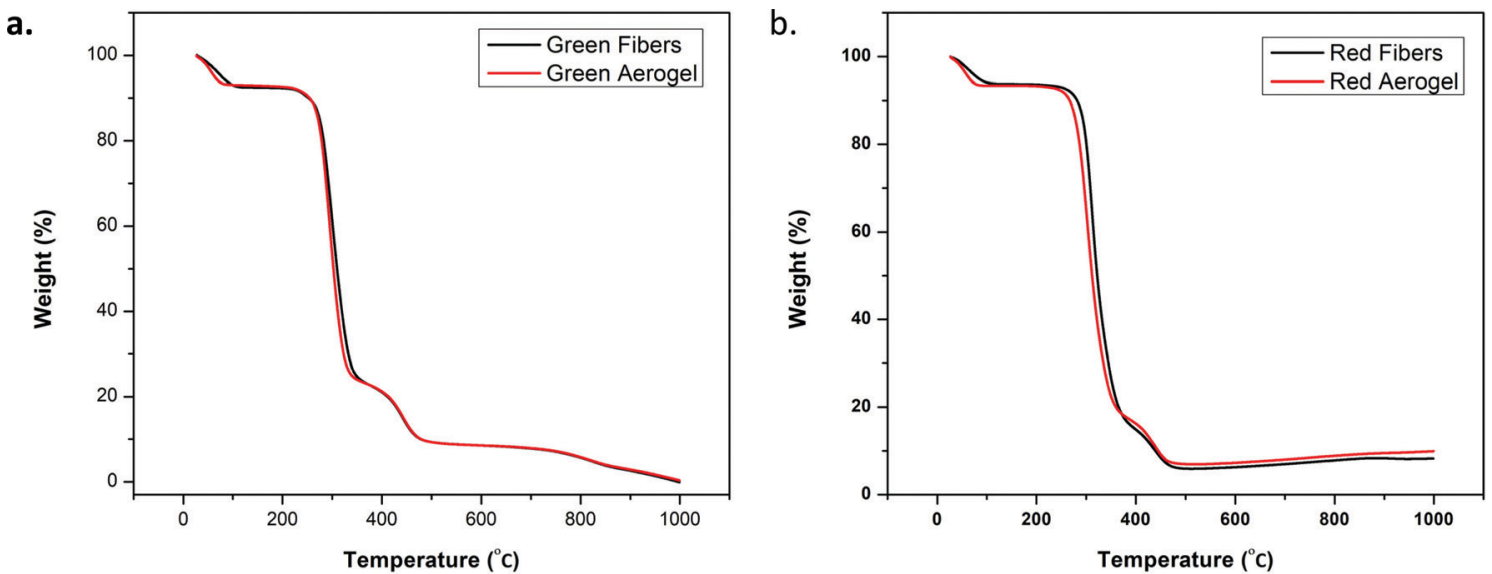

Fig. 7 (a) TGA plots for pure green fibers and the water-based aerogel; and (b) pure red fibers and the water-based aerogel.

fibers which act as the precursor of fluorescent aerogels. $\mathrm{Mn}^{2+}$ displays green to red tunable emission depending on the coordination chemistry of the doped lattice $;^{33,46}$ in this case, the green band is prevalent since high spin $\mathrm{Mn}^{2+}$ occupies low coordination tetrahedral $\mathrm{Zn}^{2+}$ sites in the doped lattice of the NRs. It is worth mentioning that the coordination chemistry and luminescence properties of the NRs remain unchanged in the fibers and in the aerogel as well. The same observation is true for the pure red emitting aerogel where the bright red emission band at $695 \mathrm{~nm}$ is ascribed to the ${ }^{2} \mathrm{E} \rightarrow{ }^{4} \mathrm{~A}_{2}$ transition due to trivalent chromium ions occupying the octahedral sites within the red nanoparticles. ${ }^{33,47,48}$ No fluorescence quenching of the fibers is observed due to the aerogel formation, rather the freeze-drying process removes the hydration from the structure which eliminates the chance of quenching. Moreover, the aerogels show higher emission intensities than fiber mats (Fig. 8b and c) due to the resultant highly organized porous wall solid-like texture that provides higher and direct interaction of light sources with the nanoparticles and therefore an efficient light absorption resulting in an enhanced luminescence for the a.

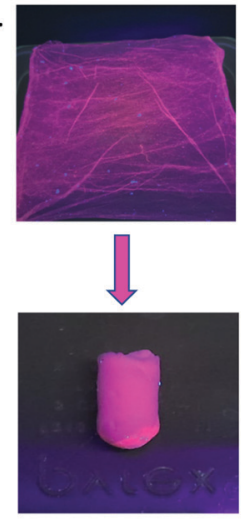

$100 \%$ red
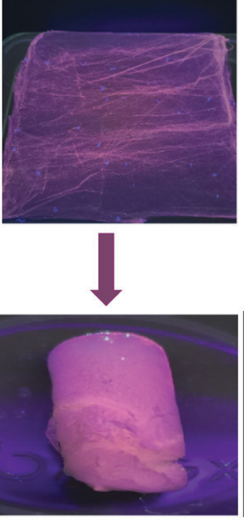

$90 \%$ red + $10 \%$ green

b.

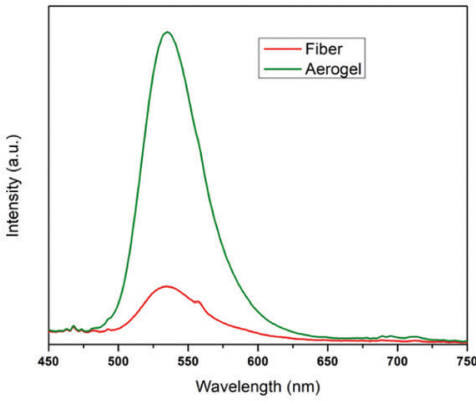

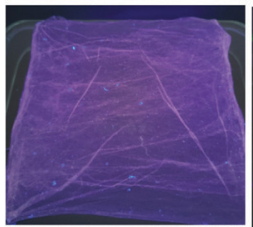

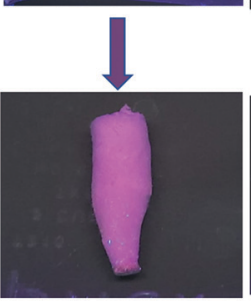

$80 \%$ red + $20 \%$ green
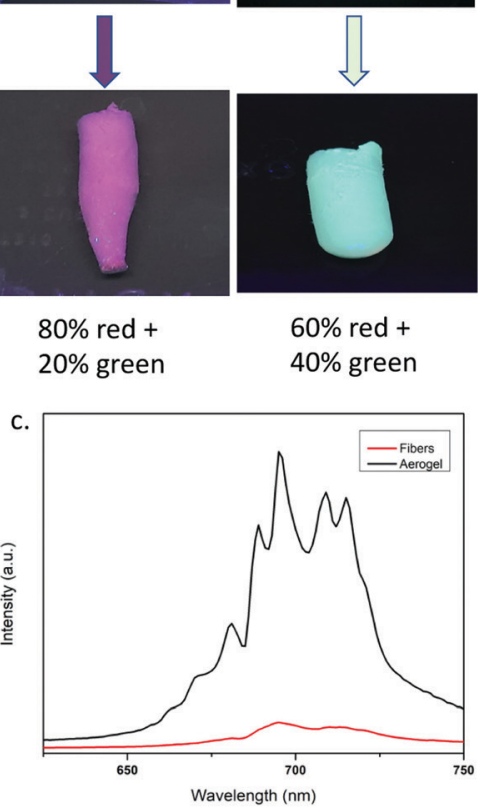
$60 \%$ red +
$40 \%$ green
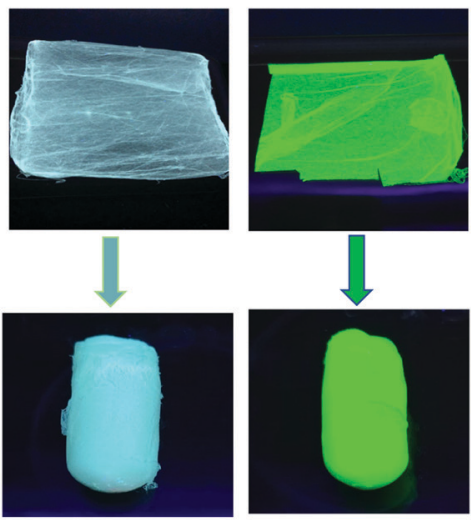

$40 \%$ red + $60 \%$ green

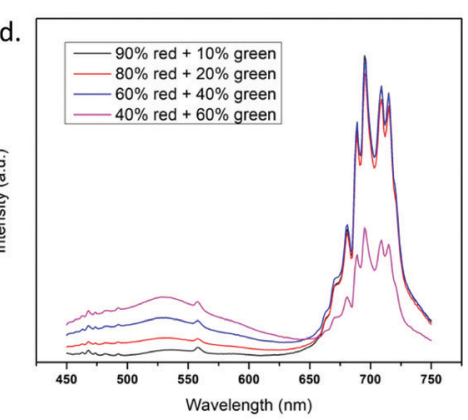

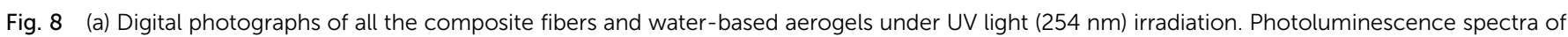
(b) green fiber and water-based aerogel; (c) red fiber and aerogel; and (d) all composite water-based aerogels. 

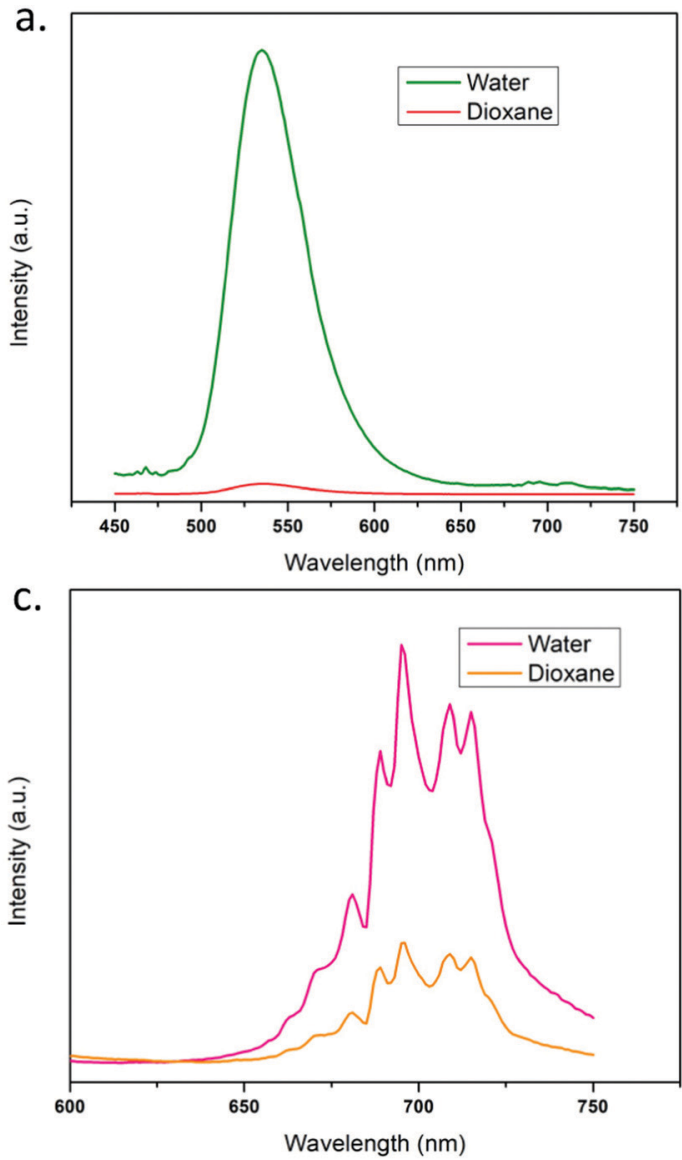

b.

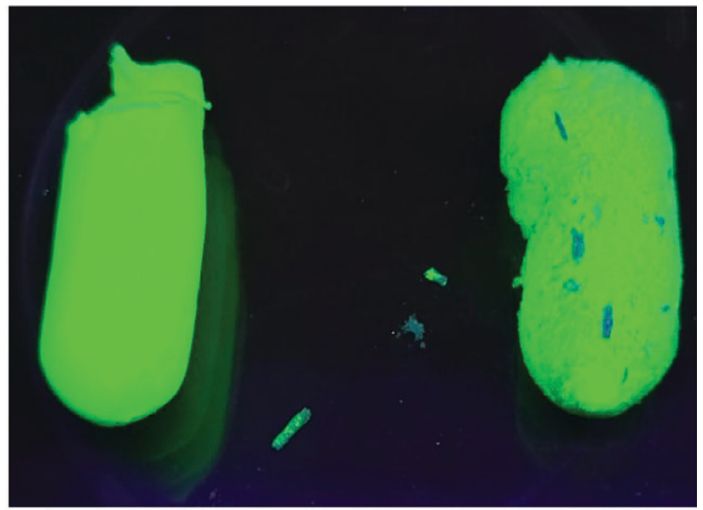

d.

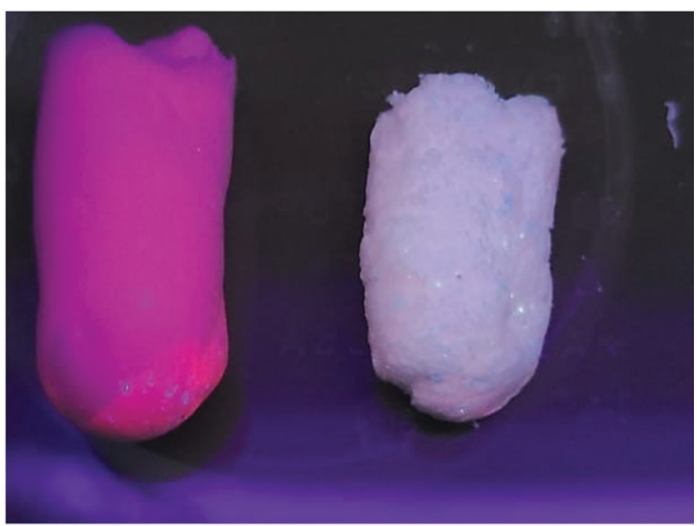

Fig. 9 Comparison of photoluminescence emission intensities of aerogels and the sponge-like 3D network using water and 1,4-dioxane as dispersion media respectively: (a) fluorescence spectra (100\% green); (b) digital photographs of the green aerogels and the sponge-like 3D network under UV light (left - water based, right - 1,4-dioxane based); (c) fluorescence spectra (100\% red); and (d) digital photographs of the red aerogels under UV light (left - water based, right - 1,4-dioxane based)

3D systems made in the water medium. However, the 3-D dioxane's fibrous network resulted in a non-uniform porous structure based on nanofiber entanglement which resulted in a higher degree of scattering and less interaction with the nanoparticles therefore causing an inefficient absorption and therefore a less luminescent system. The PL behavior of the composite aerogels is shown in Fig. 8d. All composite aerogels show visible light emission ranging from green to red depending on the ratios of green nanorods to red nanoparticles present in their precursor fine fibers which are freeze dried into aerogels. The emission spectra of the four types of composite aerogels under $254 \mathrm{~nm}$ excitation have both red and green emission peaks and their intensities are dependent on the concentration ratios of the nanoparticles present in the composite fibers from which the aerogels are formed. Fig. 9(a and c) show the comparison for the emission intensities of aerogels using water and dioxane as the dispersion medium for the synthesis. It is clearly observed that the emission intensities (both green and red) are higher for the water medium which is also depicted from the digital photographs of the aerogels taken under UV light (Fig. 9b and d). From SEM images (Fig. 4a-c), it was observed that the structures resulting from being subjected to the water medium exhibit a high degree of order, and a structure with a large surface area with homogenous dispersion of nanoparticles is obtained where light absorption is maximized. And the SEM image of the dioxane based sponge-like porous 3D network (Fig. 4d) shows the fibrous network of the nanofibers with heterogeneous pore size and distribution. The sponge-like nature of this material proved less effective in light absorption (when compared to the water-based materials), mostly due to the irregular pore size/ shape which is responsible for irregular transmission of incident light within the system and therefore reduced absorption capabilities of the encapsulated NPs/NRs, resulting in reduced photoluminescence intensity.

\section{Conclusions}

In summary, we have demonstrated water-based synthesis of ultralight color tunable luminescent aerogels/sponge-like structures from the tailored assembly of red and green emitting nanoparticles encapsulated within PVA nanofibers. Varying the concentration of ZGOM and ZGOC (manganese doped zinc 
germanate, $\mathrm{Mn}: \mathrm{Zn}_{2} \mathrm{GeO}_{4}$ and chromium doped zinc gallate, $\mathrm{Cr}: \mathrm{ZnGa}_{2} \mathrm{O}_{4}$ ) compounds promoted the development of the final colorimetric performance of the final structure. The developed study showed a significantly higher photoluminescence effect within the aerogel structure when compared to their corresponding luminescent nanofiber mats. This can be attributed to the higher surface-lower density solid 3D assembly when compared to the nanofiber mats and therefore higher and direct interaction with the nanoparticles which resulted in enhanced light absorption causing higher luminescence. Moreover, the nature of pores in the water-based aerogels is affected by the morphologies of the fillers. Notably, these aerogels/sponge-like structures were prepared by a green synthesis pathway using a toxic solvent free route (all water based). This process promotes sustainability and low toxicity within the final product which has a wide range of exciting potential applications.

\section{Conflicts of interest}

The authors declare that they have no known competing financial interests or personal relationships that could have appeared to influence the work reported in this paper.

\section{Acknowledgements}

The authors gratefully acknowledge funding from NSF under DMR PREM grant 2122178.

\section{References}

1 M. Zhang, J. Xue, Y. Zhu, C. Yao and D. Yang, ACS Appl. Mater. Interfaces, 2020, 12, 22191-22199.

2 S. Zhao, W. J. Malfait, N. Guerrero-Alburquerque, M. M. Koebel and G. Nystroem, Angew. Chem., Int. Ed., 2018, 57, 7580-7608.

3 Y. Cao, L. Lewis, W. Y. Hamad and M. J. MacLachlan, Adv. Mater., 2019, 31, 1808186.

4 L. Dou, X. Zhang, X. Cheng, Z. Ma, X. Wang, Y. Si, J. Yu and B. Ding, ACS Appl. Mater. Interfaces, 2019, 11, 29056-29064.

5 B. Cai, V. Sayevich, N. Gaponik and A. Eychmueller, Adv. Mater., 2018, 30(33), 1707518.

6 M. Aghajamali, M. Iqbal, T. K. Purkait, L. Hadidi, R. Sinelnikov and J. G. C. Veinot, Chem. Mater., 2016, 28, 3877-3886.

7 B. Wu, G. Zhu, A. Dufresne and N. Lin, ACS Appl. Mater. Interfaces, 2019, 11, 16048-16058.

8 H. Wang, Z. Shao, M. Bacher, F. Liebner and T. Rosenau, Cellulose, 2013, 20, 3007-3024.

9 F. S. Rodembusch, L. F. Campo, V. Stefani and A. Rigacci, J. Mater. Chem., 2005, 15, 1537-1541.

10 T. Mondal, S. Mondal, S. Bose, D. Sengupta, U. K. Ghorai and S. K. Saha, J. Mater. Chem. C, 2018, 6, 614-621.

11 W. Cheng, F. Rechberger and M. Niederberger, ACS Nano, 2016, 10, 2467-2475.
12 B. B. Srivastava, S. K. Gupta, S. Mohan, A. Abraham, A. Portales and Y. Mao, Mater. Lett., 2021, 297, 129964.

13 B. Gao, Y. Ma, J. Mei, S. Lu and L. Ren, Chem. Eng. Sci., 2018, 331, 597-605.

14 J. Zhu, S. Lv, T. Yang, T. Huang, H. Yu, Q. Zhang and M. Zhu, Adv. Sustainable Syst., 2020, 4, 1900141.

15 F. Deuber and C. Adlhart, Chimia, 2017, 71, 236-240.

16 W. E. Teo and S. Ramakrishna, Nanotechnology, 2006, 17, R89-R106.

17 A. Greiner and J. H. Wendorff, Angew. Chem., Int. Ed., 2007, 46, 5670-5703.

18 Y. Si, J. Yu, X. Tang, J. Ge and B. Ding, Nat. Commun., 2014, 5, 5802 .

19 G. Duan, S. Jiang, V. Jerome, J. H. Wendorff, A. Fathi, J. Uhm, V. Altstaedt, M. Herling, J. Breu, R. Freitag, S. Agarwal and A. Greiner, Adv. Funct. Mater., 2015, 25, 2850-2856.

20 S. Chabi, V. G. Rocha, E. Garcia-Tunton, C. Ferraro, E. Saiz, Y. Xia and Y. Zhu, ACS Nano, 2016, 10, 1871-1876.

21 C. Ferraro, E. Garcia-Tunon, V. G. Rocha, S. Barg, M. D. Farinas, T. E. G. Alvarez-Arenas, G. Sernicola, F. Giuliani and E. Saiz, Adv. Funct. Mater., 2016, 26, 1636-1645.

22 X. Gui, J. Wei, K. Wang, A. Cao, H. Zhu, Y. Jia, Q. Shu and D. Wu, Adv. Mater., 2010, 22, 617-621.

23 N. Kraenzlin and M. Niederberger, Adv. Mater., 2013, 25, 5599-5604.

24 F. Rechberger and M. Niederberger, Nanoscale Horiz., 2017, 2, 6-30.

25 J.-S. Kim and D. H. Reneker, Polym. Compos., 1999, 20, 124-131.

26 F. Deuber, S. Mousavi, L. Federer, M. Hofer and C. Adlhart, ACS Appl. Mater. Interfaces, 2018, 10, 9069-9076.

27 M. Thieme, S. Agarwal, J. H. Wendorff and A. Greiner, Polym. Adv. Technol., 2011, 22, 1335-1344.

28 S. Jiang, G. Duan, J. Schoebel, S. Agarwal and A. Greiner, Compos. Sci. Technol., 2013, 88, 57-61.

29 M. Langner and A. Greiner, Macromol. Rapid Commun., 2016, 37, 351-355.

30 Y. Ren, S. Wang, R. Liu, J. Dai, X. Liu and J. Yu, RSC Adv., 2016, 6, 30139-30147.

31 Y. Yu, S. Hua, M. Yang, Z. Fu, S. Teng, K. Niu, Q. Zhao and C. Yi, RSC Adv., 2016, 6, 110557.

32 B. B. Srivastava, S. K. Gupta, Y. Li and Y. Mao, Dalton Trans., 2020, 49, 7328-7340.

33 R. Barbosa, S. K. Gupta, B. B. Srivastava, A. Villarreal, H. De Leon, M. Peredo, S. Bose and K. Lozano, J. Lumin., 2021, 231, 117760.

34 B. B. Srivastava, S. K. Gupta and Y. Mao, CrystEngComm, 2020, 22, 2491-2501.

35 B. B. Srivastava, S. K. Gupta and Y. Mao, J. Mater. Chem. C, 2020, 8, 6370-6379.

36 B. B. Srivastava, A. Kuang and Y. Mao, Chem. Commun., 2015, 51, 7372-7375.

37 A. Kharazmi, N. Faraji, R. M. Hussin, E. Saion, W. M. M. Yunus and K. Behzad, Beilstein J. Nanotechnol., 2015, 6, 529-536. 
38 J. Lee, T. Isobe and M. Senna, J. Colloid Interface Sci., 1996, 177, 490-494.

39 Q. Li, X. Miao, C. Wang and L. Yin, J. Mater. Chem. A, 2015, 3, 21328-21336.

40 F. Zou, X. Hu, Q. Long, Y. Jiang, X. Xiong, Y. Qiao and Y. Huang, Nanoscale, 2014, 6, 924-930.

41 T. T. Yu, H. L. Liu, M. Huang, J. H. Zhang, D. Q. Su, Z. H. Tang, J. F. Xie, Y. J. Liu, A. H. Yuan and Q. H. Kong, RSC Adv., 2017, 7, 51807-51813.

42 D. Beke, M. V. Nardi, G. Bortel, M. Timpel, Z. Czigany, L. Pasquali, A. Chiappini, G. Bais, M. Rudolf, D. Zalka, F. Bigi, F. Rossi, L. Bencs, A. Pekker, B. G. Markus, G. Salviati, S. E. Saddow, K. Kamaras, F. Simon and A. Gali, Chem. Mater., 2021, 33, 2457-2465.
43 S. Wang, X. Zhang, Z. C. Feng and Y. Cui, Opt. Express, 2014, 22, 17440-17448.

44 M. K. Lee and S. Kang, J. Ceram. Soc. Jpn., 2018, 126, 382-388.

45 Y. Park, M. You, J. Shin, S. Ha, D. Kim, M. H. Heo, J. Nah, Y. A. Kim and J. H. Seol, Sci. Rep., 2019, 9, 3026.

46 S. K. Gupta, R. M. Kadam, R. Gupta, M. Sahu and V. Natarajan, Mater. Chem. Phys., 2014, 145, 162-167.

47 A. Bessière, S. K. Sharma, N. Basavaraju, K. R. Priolkar, L. Binet, B. Viana, A. J. J. Bos, T. Maldiney, C. Richard, D. Scherman and D. Gourier, Chem. Mater., 2014, 26, 1365-1373.

48 H. Abdou, S. K. Gupta and Y. Mao, J. Lumin., 2019, 214, 116599. 